\title{
Analytical solution of sub-harmonic nonlinear oscillation
}

\author{
Mehran Ghasempour Mouziraji ${ }^{1}$, Morteza Hoseinzadeh ${ }^{2}$, Dana Moradi ${ }^{1}$, Davood Domairy ${ }^{3}$ Mirshaban Jafari $^{2}$ \\ ${ }^{1}$ Islamic Azad University of Sari Branch, Iran \\ ${ }^{2}$ Islami Azad University Of Ayatolah Amoli Branch, Iran \\ ${ }^{3}$ Babol Noshirvani University of Technogy, Iran
}

Received: 18 October 2016, Accepted: 7 February 2017

Published online: 25 August 2017.

\begin{abstract}
The study of nonlinear oscillator is important issue in the development of theory of dynamical system. One of the newest analytical methods to solve nonlinear equations is the application of both homotopy perturbation and variational iteration techniques. Homotopy perturbation method (HPM), which does not need small parameters is compared with variational iteration method (VIM) and both of them compare with numerical solution in the field of sub-harmonic nonlinear oscillation. The justification for using this method is the difficulties and limitations of perturbation and homotopy when used individually. In this paper, homotopy perturbation method and varational iteration method are used to solve for periodic method for sub-harmonic of nonlinear oscillation. After solving the equations, we found effect of each parameter and the best value for solving equationswas $\varepsilon=0.1, \lambda=1, \alpha=0.1, \beta=1$.
\end{abstract}

Keywords: HPM, VIM, analytical solution, nonlinear oscillation, periodic solution.

\section{Nomenclature}

\begin{tabular}{|ll|ll|}
\hline$\lambda$ & Lagrange multiplayer & $\mathbf{N}$ & Nonlinear operator \\
$\varepsilon$ & SMALL parameter & $\mathbf{g}(\mathbf{X})$ & heterogeneous term \\
HPM & Homotopy perturbation method & $\mathbf{N U M}$ & Numerical method \\
VIM & Vibrational iteration method & $\mathbf{n}$ & nth approximation \\
$\mathbf{L}$ & Linear operator & $\alpha \boldsymbol{\&} \beta$ & Constant parameters \\
\hline
\end{tabular}

\section{Introduction}

Most scientific problems such as nonlinear oscillation and heat transfer are inherently of nonlinearity. We know that except a limited number of these problems, most of them do not have analytical solution. Therefore, these nonlinear equations should be solved using other methods. Some of them are solved using numerical techniques and some are solved using the analytical method of perturbation.

In the numerical method, stability and convergence should be considered so as to avoid divergence or inappropriate results. One of the methods that we used in this paper is variational iteration method. This method is based on the use of lagrange multipliers for identification of optimal values of parameters in a functional. Using this method a rapid convergent sequence is produced. Therefore, many different methods have recently introduced some ways to eliminate the small parameter, such as artificial parameter method introduced by He [1,2,3], the homotopy analysis method by Liao $[3,4]$, the variational iteration method by $\mathrm{He}$ [5-7]. One of the semi-exact methods is the homotopy perturbation method 
[8-15]. The applications of this method in different fields of nonlinear equations such as oscillator, integro-differential equations, Laplace transform, fluid mechanics and heat transfer have been studied by Cai [16], Cveticanin [17], El-Shahed [18], Abbas- bandy [19], Siddiqui [20,21] and Ganji [22-25].Recently, several researchers simulated various problems by means of new numerical and analytical methods [26-32].

In this paper we apply HPM and VIM for periodic solution of sub-harmonic nonlinear oscillation equation in the form.

$$
X+\left(1+\varepsilon \cos (\lambda t)\left[\alpha X+\beta X^{3}\right]=0\right.
$$

and boundary conditions for this equation are

$$
X(0)=X_{0}, X^{\prime}(0)=0
$$

\section{Basic idea of homotopy perturbation method}

In this paper, we apply the homotopy perturbation method to solve this equation. To illustrate the basic ideas of the new method, we consider the following nonlinear differential equation,

$$
A(u)-f(r)=0, r \in \Omega
$$

with boundary conditions of

$$
B\left(u, \frac{\partial u}{\partial n}\right)=0, r \in \Gamma
$$

where $A(u)$ is defined as follows

$$
A(u)=L(u)+N(u)
$$

where $L$ stands for the linear and $N$ for the nonlinear part. Homotopy perturbation structure is shown as the following equation.

$$
p^{2}: X_{2}^{\prime \prime}(t)+0.1 \cos (t) X_{1}(t)^{2}+X_{2}(t)+0.03 \cos (t) X_{0}(t)^{2} X_{1}(t)+0.3 X_{0}(t)^{2} X_{1}(t)=0
$$

where

$$
X(r, p): \Omega \times[0,1] \rightarrow R
$$

Obviously, using Equation . (4) we have

$$
\begin{aligned}
& H(X, 0)=L(X) \cdot L\left(u_{0}\right)=0 \\
& H(X, 1)=A(X) \cdot f(r)=0 \\
& H(X, 1)=A(X) \cdot f(r)=0
\end{aligned}
$$


where $p \in[0,1]$ is an embedding parameter and $\mathrm{u}_{0}$ is the first approximation that satisfies the boundary condition. The process of changes in $p$ from zero to unity is that of $X(r, p)$ changing from $u_{0}$ to $u(r)$. We consider $X$, as the following.

$$
X=X_{0}+p X_{1}+p^{2} X^{2}
$$

and the best approximation for the solution is:

$$
u=\lim _{p \rightarrow 1} X=X_{0}+X_{1}+X_{2}+\ldots
$$

The above convergence is discussed in $[16,17]$.

\section{Application of HPM to solve this problem}

In Equation (9), assuming $\varepsilon=0.1, \lambda=1, \alpha=0.1, \beta=1$ and separating the linear and nonlinear parts of the equation and apply homotopy perturbation method and substituting Equation (7) into Eq (4) and this equation is created.

$$
\begin{aligned}
H(X, P): & =(1-p)\left(\frac{d^{2}}{d t^{2}} X_{\circ}(t)+p\left(\frac{d^{2}}{d t^{2}} X_{1}(t)\right)+p^{2}\left(\frac{d^{2}}{d t^{2}} X_{2}(t)\right)\right. \\
& +p^{3}\left(\frac{d^{2}}{d t^{2}} X_{3}(t)\right)+X_{0}(t)+p X_{1}(t)+p^{2} X_{2}(t)+p^{3} X_{3}(t) \\
& +p\left(\frac{d^{2}}{d t^{2}} X_{3}(t)+p\left(\frac{d^{2}}{d t^{2}} X_{1}(t)\right)+p^{2}\left(\frac{d^{2}}{d t^{2}} X_{2}(t)\right)\right. \\
& +p^{3}\left(\frac{d^{2}}{d t^{2}} X_{3}(t)\right)+(1+0.1 \cos (t))\left(X_{0}(t)+p X_{1}+p^{2} X_{2}(t)+p^{3} X_{3}(t)+0.1\left(X_{0}(t)\right)+p^{2} X_{2}(t)+p^{3} X_{3}(t)^{3}\right.
\end{aligned}
$$

In this step we collect and expand equation (11) based on $p$-terms and find and solve the theirs multipliers.

$$
\begin{aligned}
& p^{0}: X_{0}^{\prime \prime}(t) X_{0}(t)=0 \\
& X_{0}(t):=\cos (t) \\
& p^{1}: X_{1}^{\prime \prime}(t)+0.1 \cos (t)^{2}+0.01 \cos (t)^{4}+X_{1}(t)+0.1 \cos (t)^{3}=0 \\
& X_{1}(t):=0.022833 \cos (t)+0.000666 \cos (t)^{4}+0.036 \cos (t)^{2}-0.072+0.0125 \cos (t)^{3}-0.0375 \sin (t) t \\
& p^{2}: X_{2}^{\prime \prime}(t)+0.1 \cos (t) X_{1}(t)^{2}+X_{2}(t)+0.03 \cos (t) X_{0}(t)^{2} X_{1}(t)+0.3 X_{0}(t)^{2} X_{1}(t)=0 \\
& X_{2}(t):=0.003545 \cos (t)+0.000075 \cos (t)^{3} \sin (t) \\
& -0.014062 \cos (t)^{2} \sin (t) t-0.0014 \cos (t) \sin (t) t+0.00001 \sin (t) t+0.006776 \\
& +4.16666610^{-7} \cos (t)^{7}+0.00001642857143 \cos (t)^{6}+0.000204 \cos (t)^{5}+0.000841 \cos (t)^{4} \\
& +0.000493 \cos (t)^{3}-0.004788 \cos (t)^{2}-0.000703 \cos (t) t^{2}
\end{aligned}
$$

So $X$ will be generally as follows.

$$
\begin{aligned}
& X(t):=1.019288 \cos (t)+0.001508 \cos (t)^{4} \\
& +0.031211 \cos (t)^{2}-0.065223+0.012993 \cos (t)^{3}-0.037489 \sin (t) t \\
& -0.000075 \cos (t)^{3} \sin (t) t-0.001406 \cos (t)^{2} \sin (t) t-0.0014 \cos (t) \sin (t)+4.166666 \\
& +0.000016 \cos (t)^{6}+0.000204 \cos (t)^{5}-0.000703 \cos (t) t^{2}
\end{aligned}
$$


In this step, after solving by HPM method we compare it with numerical solution and this result is shown figure (1).

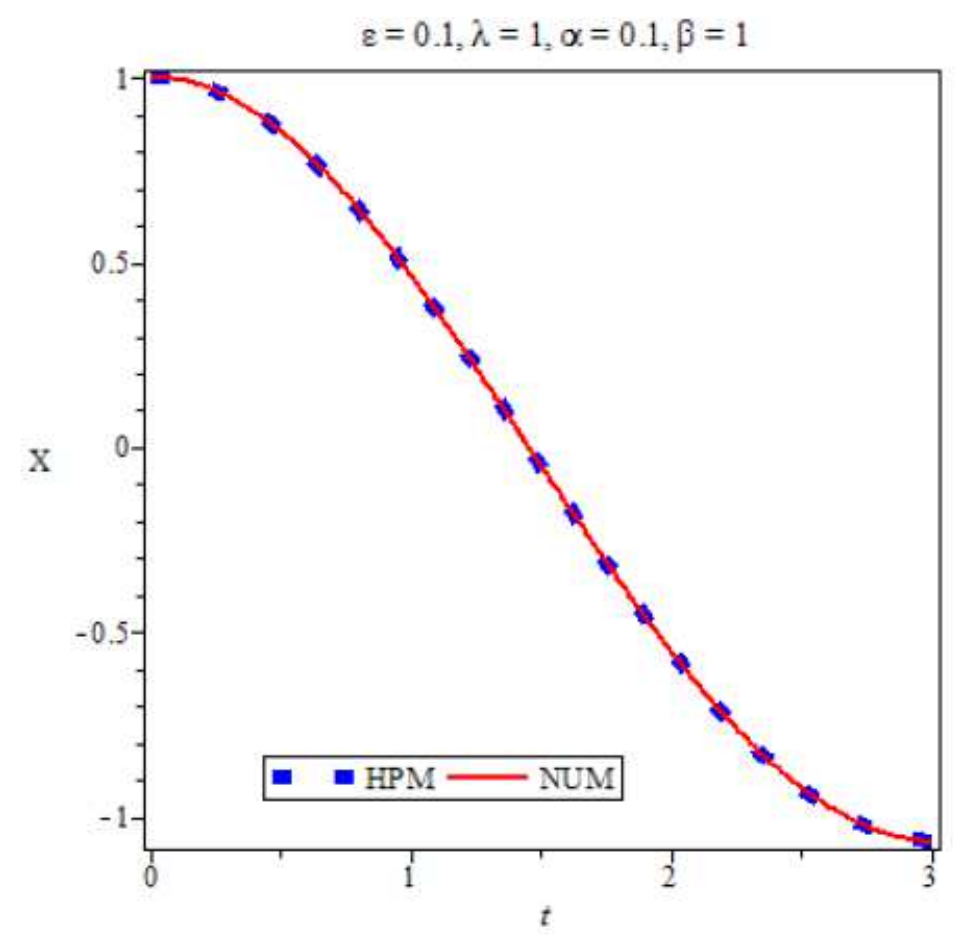

Fig. 1: omparison of HPM and NUM.

\section{The basic idea of variational iteration method}

To illustrate the basic concepts of the VIM $[8,9,13]$, we consider the following deferential equation

$$
L X+N X=g(X)
$$

where $L$ is a linear operator, $N$ a nonlinear operator, and $g(X)$ a heterogeneous term. According to the VIM, we can construct a correction functional as follows. VIM is so powerful method to solve nonlinear equation.

\section{Application of (VIM) to solve this problem}

To solve equation (9) and (10), we have the correction functional as

$$
X_{(n+1)}=X_{n}(t)+\int_{0}^{t} \lambda\left(\frac{d^{2} X_{(n)} \tau}{d \tau^{2}}+(1+0.1 \cos (\tau))\left(X_{n}(\tau)+0.1 X_{n}(\tau)^{3}\right)\right) d \tau
$$

The $\lambda$ multiplier is

$$
\operatorname{Sin}(t)
$$


Substituting equation (17) into the correction functional system (16) result iteration formula and create the first iteration follow as

$$
X_{1}(t):=X_{0}(t)+\int_{0}^{t} \lambda\left(\frac{d^{2}}{d \tau^{2}} X_{n}(\tau)+(1+0.1 \cos (\tau))\left(X_{n}(\tau)+0.1 X_{n}(\tau)^{3}\right)\right) d \tau
$$

After substituting $X_{0}$ in the equation (18) we find $X_{1}(t)$.

$$
X_{1}(\tau):=X_{n}(t)+\int_{0}^{t}-\sin (-\tau+t)\left(\frac{d^{2}}{d \tau^{2}} X_{0}(\tau)+(1+0.1 \cos (\tau))\left(X_{0}(\tau)+0.1 X_{0}(\tau)^{3}\right)\right) d \tau,
$$

and value of $X_{1}(t)$ is

$$
\begin{aligned}
X_{1}(\tau): & =1.032208 \cos (\tau)+0.003125 \cos (3 . \tau)-0.05375-0.0375 \sin (\tau) \tau \\
& +0.018333 \cos (2 . \tau)+0.000083 \cos (4 . \tau) .
\end{aligned}
$$

The second iteration

$$
X_{2}(t):=X_{1}(t)+\int_{0}^{t}\left(-\sin (-\tau+t)\left(\frac{d^{2}}{d \tau^{2}} X_{1}(\tau)+(1+0.1 \cos (\tau))\left(X_{1}(\tau)+0.1 X_{1}(\tau)^{3}\right)\right) d \tau\right.
$$

After substituting $X_{1}$ in the equation (18) we find $X_{2}(t)$.

$$
\begin{aligned}
X_{2}(\tau): & =0.000017 \sin (\tau) \tau^{3}-0.000773 \cos (\tau) \tau^{2}-4.94384710^{-7} \cos (\tau) \tau^{4} \\
& -1.77429110^{-8} \tau^{2} \cos (5 . \tau)-7.21958710^{-1 \circ} \tau^{2} \cos (6 . \tau)-0.000013 \tau^{2} \cos (3 . \tau) \\
& -9.15527310^{-12} \tau^{2} \cos (2 . \tau)-5.18701910^{-11} \tau \sin (8 . \tau)-4.96937410^{-8} \tau \sin (6 . \tau) \\
& -9.95843810^{-7} \tau \sin (5 . \tau)-1.585194910^{-9} \tau \sin (7 . \tau)-1.1596679610^{-12} \tau \sin (9 . \tau) \\
& -9.86426710^{-15} \tau \sin (10 . \tau)-0.00053 \tau \sin (2 . \tau)-4.39453110^{-8} \tau^{3} \sin (2 . \tau) \\
& -4.82490210^{-7} \tau^{2} \cos (4 . \tau)+4.39453110^{-9} \tau^{3} \sin (4 . \tau)-0.000016 \tau \sin (4 . \tau) \\
& -0.00035 \tau \sin (3 . \tau)+1.64794910^{-7} \tau^{3} \sin (3 . \tau)+0.000007 \tau^{2} \\
& +0.000014 \cos (5 . \tau) 8.22776410^{-7} \cos (6 . \tau)+3.81100910^{-8} \cos (7 . \tau) \\
& +5.98625310^{-11} \cos (9 . \tau)+4.30583110^{-18} \cos (13 . \tau)+1.73032810^{-12} \cos (10 . \tau) \\
& +4.29792910^{-14} \cos (11 . \tau)+1.72139110^{-9} \cos (8 . \tau) 6.70264310^{-16} \cos (12 . \tau) \\
& -0.038185 \sin (\tau) \tau-0.04866+0.003287 \cos (3 . \tau)+0.016509 \cos (2 . \tau) \\
& +0.00019 \cos (4 . \tau)+1.028658 \cos (\tau),
\end{aligned}
$$

and finally find third iteration 


$$
\begin{aligned}
X_{1}(t): & =1.17280110^{-24} t^{10}-6.38179910^{-24} t^{5} \sin (15 . t)+2.50927410^{-37}+1.63971410^{-16} t^{6} \cos (7 . t) \\
& -5.97830610^{-30} t^{5} \sin (19 . t)-2.30094710^{-35} t^{2} \cos (26 . t)+1.24695710^{-22} t^{11} \sin (5 . t) \\
& +6.24068810^{-59} \cos (40 . t)+8.9441510^{-25} t^{3} \sin (18 . t)+6.64843610^{-24} t^{7} \sin (12 . t) \\
& +1.65412510^{-25} \cos (21 . t)+2.28313910^{-18} t^{10}+305714552310^{-28} t^{7} \sin (15 . t) \\
& -1.53650110^{-22} t^{5} \sin (14 . t)+3.31501110^{-8} t^{4}+0.000011 \sin (t) t^{3}-0.000749 \cos (t) t^{2} \\
& -1.3151210^{-7} \cos (t) t^{4}+4.48330310^{-15} t^{8}+1.07356610^{-32} \cos (26 . t)+3.28901310^{-31} \cos (25 . t) \\
& -1.34447810^{-10} t^{5} \sin (2 . t)-7.52824210^{-10} t^{11} \sin (2 . t)-7.83363710^{-27} t^{5} \sin (17 . t) \\
& +7.74381510^{-54} \cos (38 . t)+3.28969610^{-34} \cos (27.7)+8.41919110^{-46} \cos (34 . t) \\
& +1.19287710^{-51} \cos (37 . t)+6.71338110^{-27} \cos (22 . t)+1.48614810^{-17} \cos (15 . t) \\
& +9.42220910^{-36} \cos (28 . t)+2.85026710^{-42} \cos (32 . t)+3.8958110^{-20} \cos (17 . t) \\
& +3.19712810^{-56} \cos (39 . t)+5.22316210^{-44} \cos (33 . t)+8.7381910^{-23} \cos (19 . t) \\
& +2.74365710^{-16} \cos (14 . t)+4.53133810^{-22} t^{12}+7.26600610^{-12} t^{6}+6.53940910^{-22} t^{7} \sin (10 . t) .
\end{aligned}
$$

In this step we compare result of VIM and numerical method which shows in figure (2)

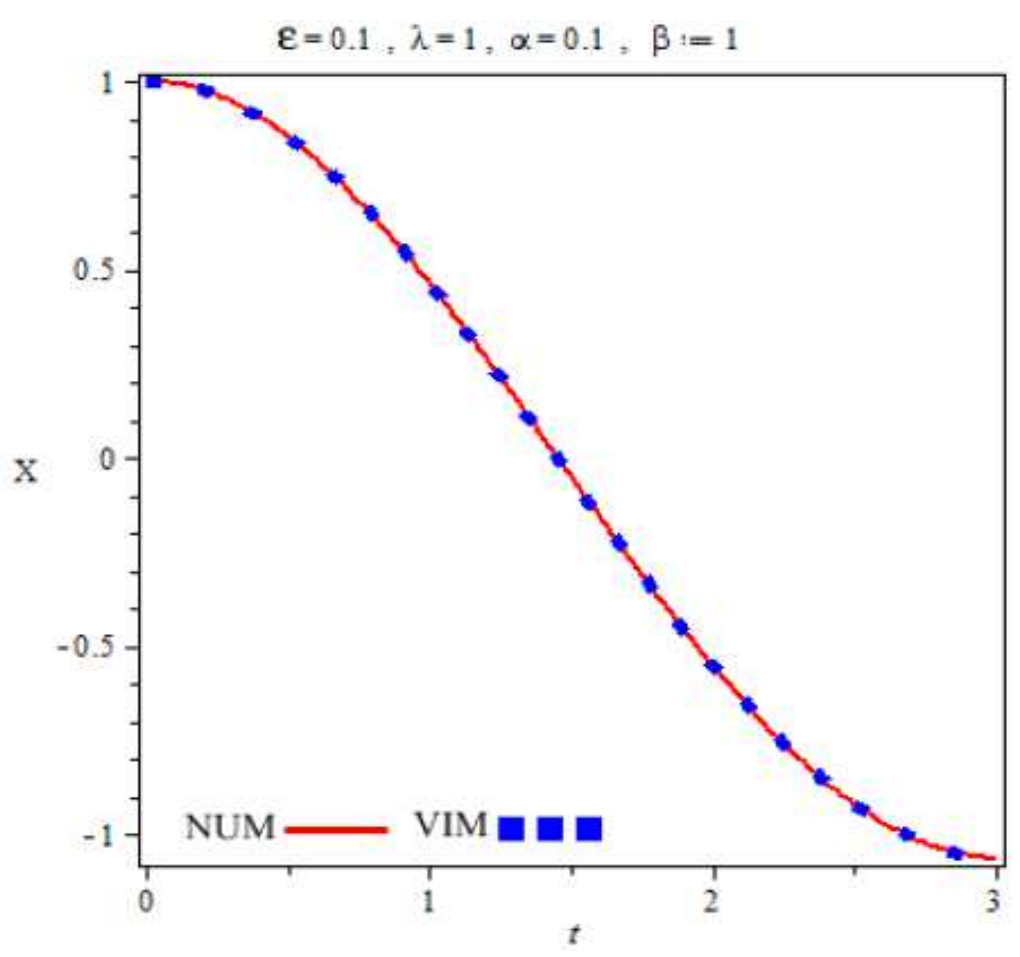

Fig. 2: Comparison of VIM and NUM. 

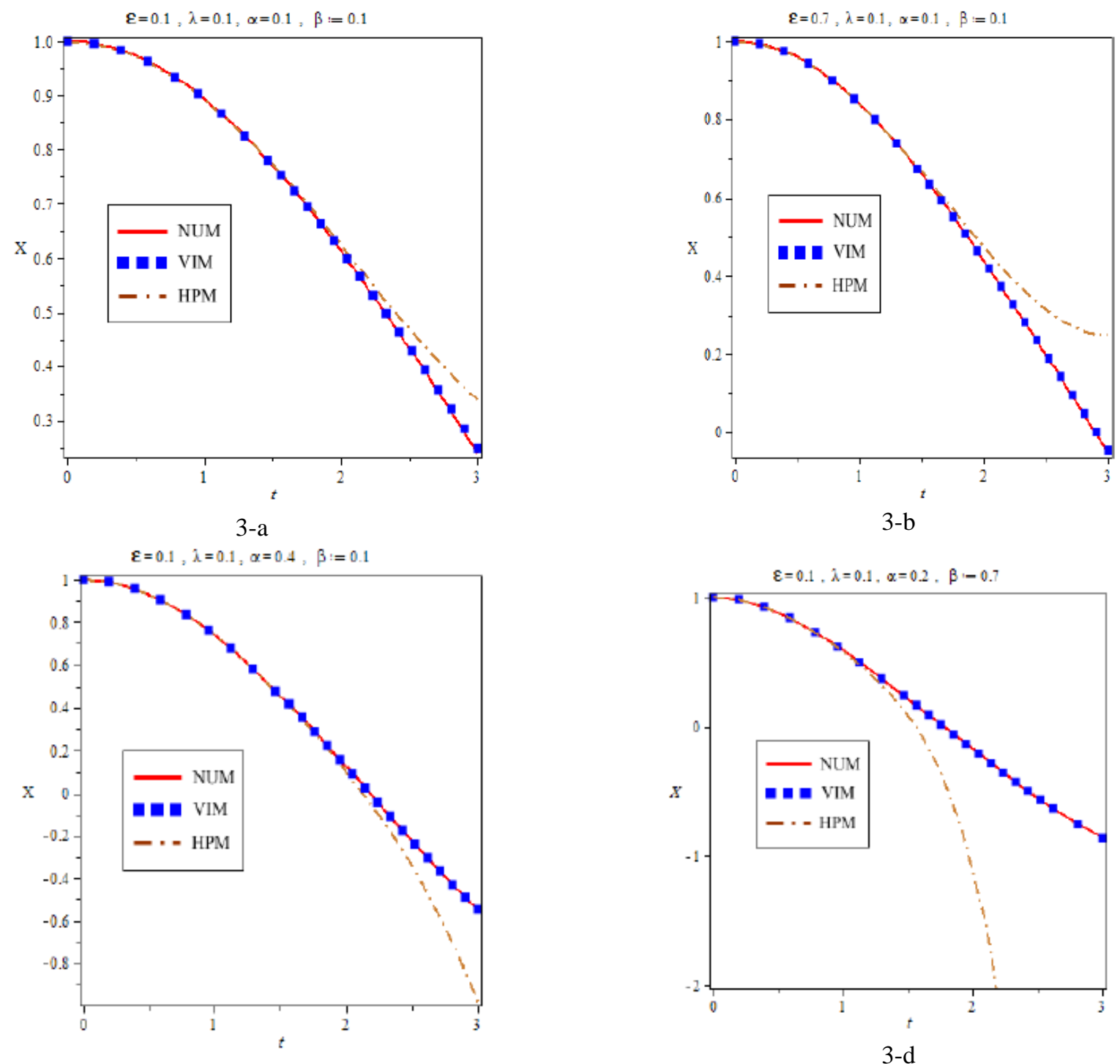

3-c

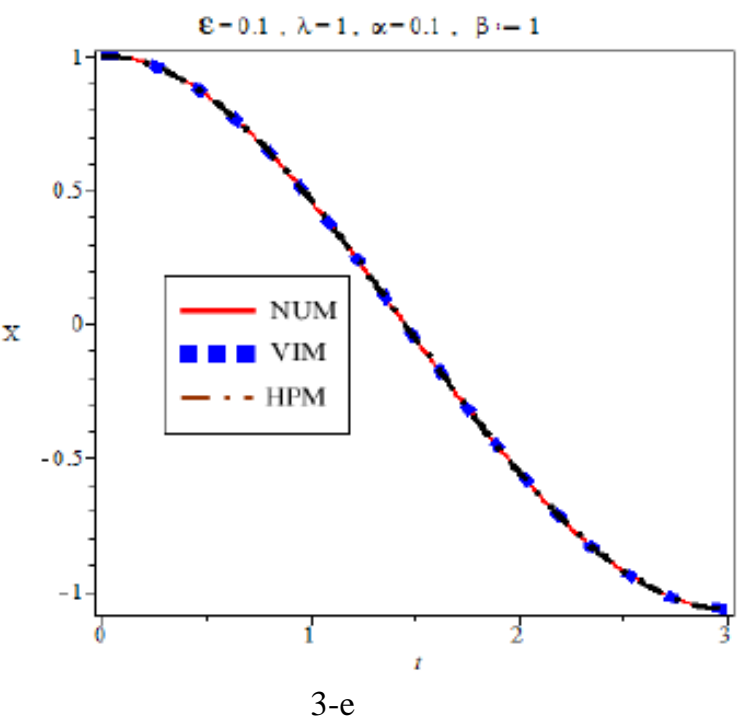

Fig. 3: Comparison of results. 


\section{Conclusion}

In this paper we use HPM and VIM and numerical method for periodic solution of sub-harmonic of non- linear oscillation. Finally it was found that the equation we understand the HPM method have less computing than VIM method but the result of both method are the same numerical solution. Figure (3) shows the comparison of result. Also, Effects of active parameters on $X$ are shown in Figs. (4-6). Increasing $\alpha$ leads to decrease $X$. In addition, minimum point is shift to lower time with increase of $\alpha$. Similar trend is observed for $\beta$.X profile decreases with increase of $\varepsilon$. $\lambda$ has no significant effect of $X$.

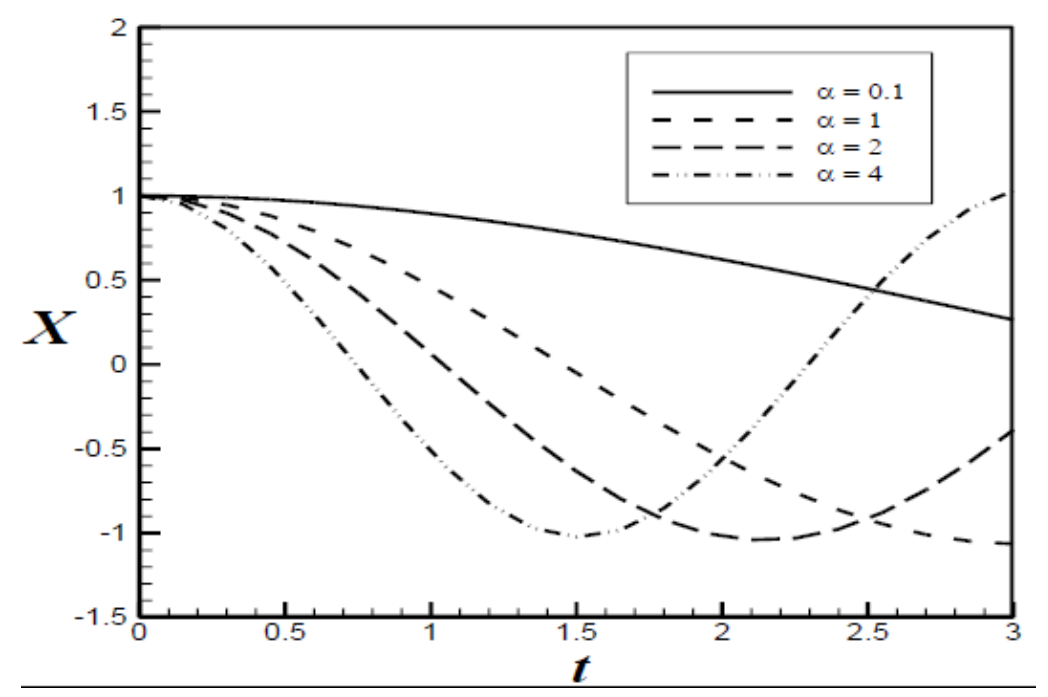

Fig. 4: Effect of $\alpha$ on $X$ when $\varepsilon=0.1, \beta=0.1, \lambda=1$.

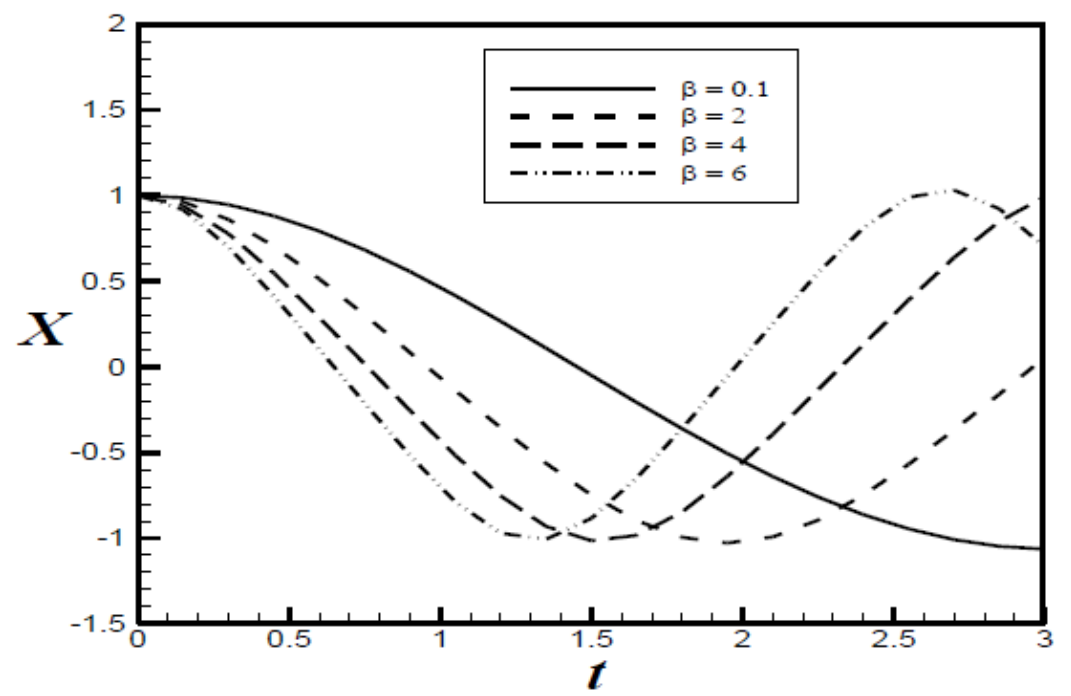

Fig. 5: Effect of $\beta$ on $X$ when $\varepsilon=0.1, \lambda=1, \alpha=1$. 


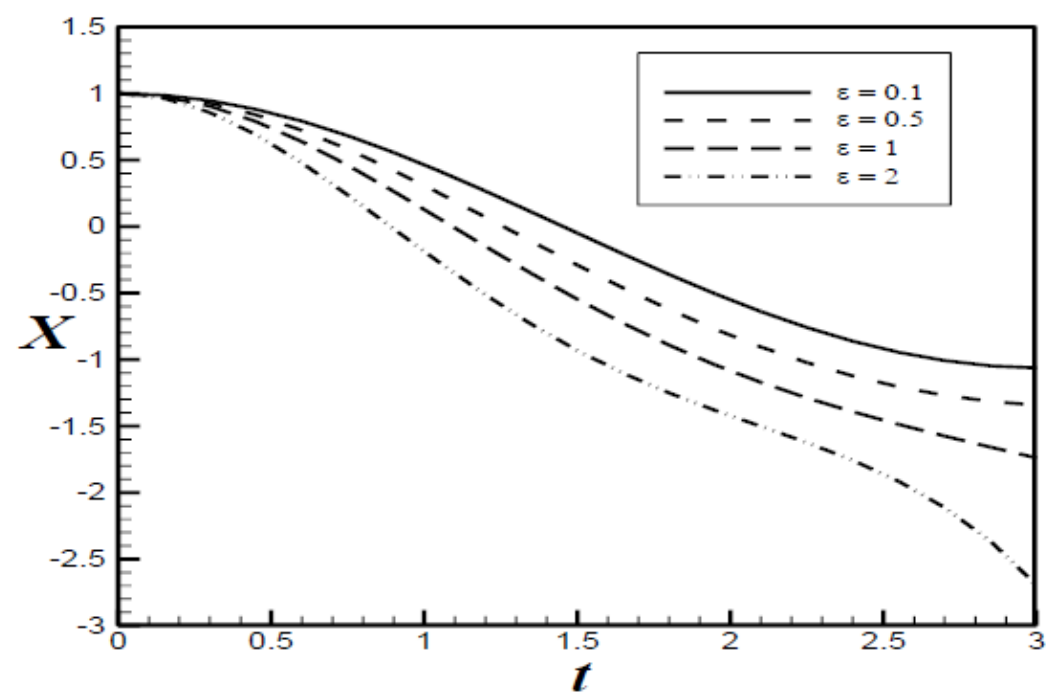

Fig. 6: Effect of $\varepsilon$ on $X$ when $\beta=0.1, \lambda=1, \alpha=1$.

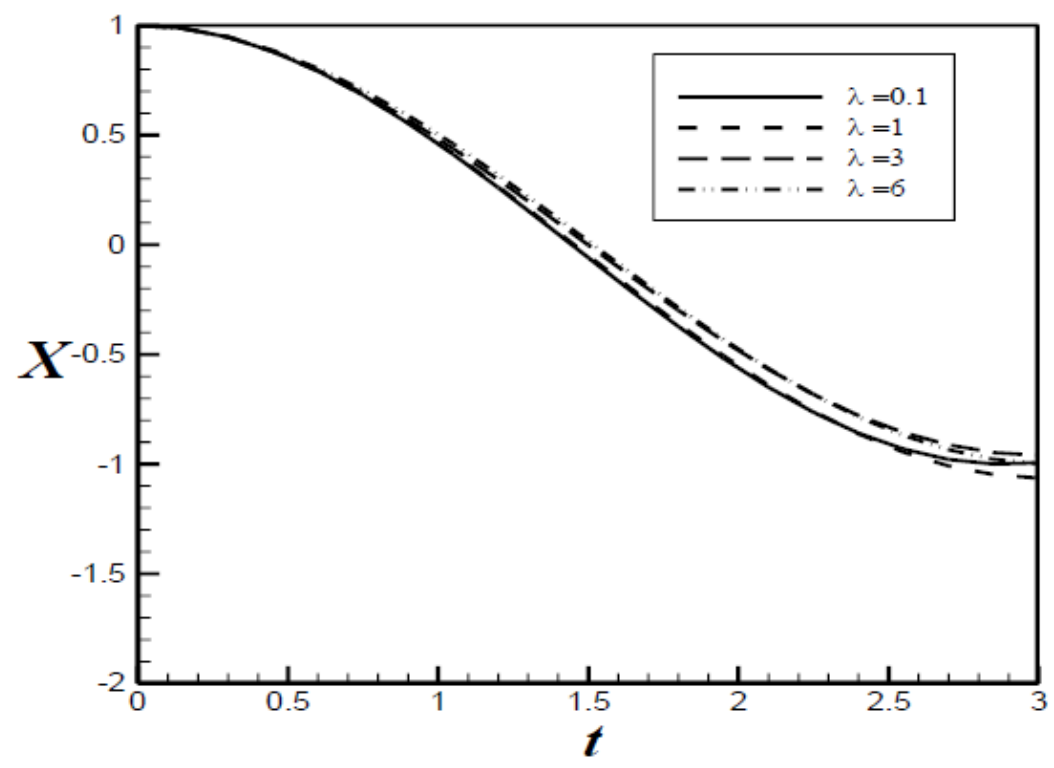

Fig. 7: Effect of $\lambda$ on $X$ when $\beta=0.1, \varepsilon=0.1, \alpha=1$. 


\section{Competing interests}

The authors declare that they have no competing interests.

\section{Authors' contributions}

All authors have contributed to all parts of the article. All authors read and approved the final manuscript.

\section{References}

[1] J.H. He, dissertation .de-verlag im internet $\mathrm{GmbH}, 2006$.

[2] J.H. He, Int. J. Mod. Phys. B 20 (10) (2006) 1141.

[3] A. Rajabi , D.D. Ganji, H. Taherian, Phys. Lett.360 (2) (2007) 131.

[4] S.J. Liao, Int. J. Non-Linear Mech. 303 (1995) 371.

[5] S.J. Liao, Eng. Anal. Bound. Elm. 202 (1997) 91.

[6] J.H. He, J. Comput. Math. Appl. Mech. Eng. 167 (1998) 57.

[7] J.H. He, Comput. Math. Appl. Mech. Eng. 167 (1998) 69.

[8] J.H. He, Int. J. Non-Linear Mech. 344 (1999) 699.

[9] J.H. He, J. Comput. Math. Appl. Mech. Eng. 17 (8) (1999) 257.

[10] J.H. He, Int. J. Non-Linear Mech. 351 (2000) 37.

[11] J.H. He, Phys. Lett. A 350 (1-2) (2006) 87.

[12] J.H. He, X.H. Wu, Chaos Solitons Fractals 29 (1) (2006) 108.

[13] J.H. He, Phys. Lett. A 347 (4-6) (2005) 228.

[14] J.H. He, Chaos Solitons Fractals 26 (3) (2005) 827.

[15] J.H. He, Chaos Solitons Fractals 26 (3) (2005) 695.

[16] J.H. He, Int. J. Non-Linear Sci. Numer. Simul. 6 (2) (2005) 207.

[17] X.C. Cai, W.Y. Wu, M.S. Li, Int. J. Non-Linear. Sci. Numer. Simul. 7 (1) (2006) 109.

[18] L. Cveticanin, Chaos Solitons Fractals 30 (5) (2006) 1221.

[19] M. El-Shahed, Int. J. Non-Linear Sci. Numer. Simul. 6 (2) (2005) 163.

[20] S. Abbasbandy, Chaos Solitons Fractals 30 (5) (2006) 1206.

[21] A.M. Siddiqui, R. Mahmood, Q.K. Ghori, Int. J. Non-Linear Sci. Numer.Simul. 7 (1) (2006) 7.

[22] A.M. Siddiqui, M. Ahmed, Q.K. Ghori, Int. J. Non-Linear Sci. Numer. Simul. 7 (1) (2006) 15.

[23] D.D. Ganji, M. Rafei, Phys. Lett. A 356 (2) (2006) 131.

[24] D.D. Ganji, A. Rajabi, Int. Commun. Heat Mass Transfer 33 (3) (2006) 391.

[25] Mohsen Sheikholeslami, Magnetic field influence on nanofluid thermal radiation in a cavity with tilted elliptic inner cylinder, Journal of Molecular Liquids, Journal of Molecular Liquids 229 (2017) 137-147.

[26] M. Sheikholeslami, D.D. Ganji, Transportation of MHD nanofluid free convection in a porous semi annulus using numerical approach, Chemical Physics Letters 669 (2017) 202-210.

[27] M. Sheikholeslami, K. Vajravelu, Nanofluid flow and heat transfer in a cavity with variable magnetic field, Applied Mathematics and Computation, 298 (2017) 272-282.

[28] Mohsen Sheikholeslami, Influence of Lorentz forces on nanofluid flow in a porous cylinder considering Darcy model, Journal of Molecular Liquids 225 (2017) 903-912.

[29] Mohsen Sheikholeslami, CVFEM for magnetic nanofluid convective heat transfer in a porous curved enclosure, Eur. Phys. J. Plus (2016) 131: 413, DOI: 10.1140/epjp/i2016-16413-y.

[30] M. Sheikholeslami, Houman B. Rokni, Nanofluid two phase model analysis in existence of induced magnetic field, International Journal of Heat and Mass Transfer 107 (2017) 288-299.

[31] M. Sheikholeslami, S.A. Shehzad, Magnetohydrodynamic nanofluid convection in a porous enclosure considering heat flux boundary condition, International Journal of Heat and Mass Transfer 106 (2017) 1261-1269. 